\title{
UPPER-LIMB EXOSKELETON FOR HUMAN MUSCLE FATIGUE
}

\author{
S. K. ALI* \\ Autmomatic Control and System Engineering, University of Sheffield, \\ Sheffield, United Kingdom \\ *E-mail: skali1@sheffield.ac.uk \\ M. O. TOKHI \\ School of Engineering, London South Bank University \\ London, United Kingdom
}

\begin{abstract}
Human muscle fatigue is identified as one of the causes to musculuskeletal disorder (MSD). The objective of this paper is to investigate the effect of an exoskeleton in dealing with muscle fatigue in a virtual environment. The focus of this work is, for the exoskeleton to provide support as needed by human joint. A (Proportional, Integration and Derivative) controller is used for both human and exoskeleton. Simmechanics and Simulink are used to evaluate the performance of the exoskeleton. Experiments show that human is able to maintain doing the job by wearing the exoskeleton.
\end{abstract}

Keywords: Predictive fatigue model; upper-limb exoskeleton; virtual environment.

\section{Introduction}

Muscle fatigue is a well-known problem to human. Many factors could be contributed to human fatigue such as heavy physical activities, repetitive works, dealing with above-head work and duration of exposure to working environment. Muscle fatigue could effect human's quality of life and performance degradation. In a worse scenario, fatigue could lead to musculuskeletal disorder (MSD). There are two sectors that could lead human to critical fatigue: industry and rehabilitation.

Exoskeleton is one of the solution to solve human fatigue problem. Several works have been done such as in. ${ }^{1,2}$ Most current works related to muscle fatigue and exoskeleton use electromyography (EMG) signal to identify the fatigue. Another ways to measure fatigue is by adding the fatigue model 
to the developed muscle model. ${ }^{2-4}$ However, a limitation to the second approach is, it is difficult to acquire an accurate force distribution for each individu muscle. ${ }^{4,5}$ Hence, ${ }^{5}$ proposed a new fatigue model which using an information from joint level. The torque of human joint could be acquired using inverse dynamics. Due to this reason, an investigation is done to see the effect of joint fatigue model developed by Ma et.al (2009) to human joint and to evaluate the performance of an exoseleton in dealing with human fatigue during movements.

To achieve the investigation, the joint fatigue model developed by ${ }^{5}$ is applied to human joint. Then, an algorithm is developed to activate the exoskeleton using the reading from human joint. In the Section (2), the joint fatigue model is introduced, and an observation is done. Then, in the Section (3), the performance of the exoskeleton with human joint fatigue model is presented.

\section{Human Joint Fatigue Model}

As mention in Section (1), joint fatigue model by ${ }^{5}$ is used. The joint fatigue model is an extension from muscle fatigue model, which also developed by Ma et. al. Initially, Ma et. al developed fatigue index which presented as in Eq.(1). The basis new fatigue muscle model is presented in Eq.(2). Further explanation could be referred to. ${ }^{4}$

$$
\begin{gathered}
\frac{d U(t)}{d t}=\frac{M V C}{F_{\text {cem }}(t)} \frac{F_{\text {load }}}{F_{\text {cem }}(t)} \\
\frac{d F_{\text {cem }}(t)}{d t}=-k \frac{F_{\text {cem }}(t)}{M V C} F_{\text {load }}(t) \\
\frac{d \tau_{\text {cem }}(t)}{d t}=-k \frac{\tau_{\text {cem }}(t)}{\tau_{M V C}} \tau_{\text {joint }}(t)
\end{gathered}
$$

Since this paper is applying joint fatigue model, thus Eq. (2) could be rewriten as in Eq. (3). The definition for each term in Eq.(1), (2) and (3) are presented in Table (1). Simulink is used as a platform to apply the joint fatigue model and Simmechanic is used as a platform for human simulation.

Fig. (1) shows human fatigue model system. As shown in Fig. (1)(a), there are three main blocks: fatigue model, human-exoskeleton and performance. The fatigue model block contains Eq.(3). Fig.(1)(b) shows an 
Table 1. Parameters in muscle and joint fatigue model. ${ }^{4,5}$

\begin{tabular}{ccc}
\hline Muscle-fatigue Model & Unit & Definition \\
\hline MVC $/ \tau_{\text {max }}$ & Newton(N) & Muscle Maximum voluntary contraction \\
$F_{\text {cem }} / \tau_{\text {cem }}$ & Newton(N) & Muscle force capacity at time instant $t$ \\
$F_{\text {load }} / \tau_{\text {load }}$ & Newton(N) & Muscle load at time instant $t$ \\
\hline Joint-fatigue Model & Unit & Definition \\
\hline$\tau_{\text {max }}$ & Newton meter $(\mathrm{Nm})$ & Maximum joint strength \\
$\tau_{\text {cem }}$ & Newton meter $(\mathrm{Nm})$ & Joint strength at time instant $t$ \\
$\tau_{\text {load }}$ & Newton meter $(\mathrm{Nm})$ & Torque load at time instant $t$ \\
\hline$k$ & $\min ^{-1}$ & Fatigue rate \\
$t$ & min & Time \\
\hline
\end{tabular}

integration between the joint fatigue to human model. Revolute joint is used to present human joint. Thus, it is essential to have a controller to control the joint. In this paper, conventional PID controller is used for the shoulder joint. A conventional PID controller is chosen because of its easyness in tuning the parameter. The parameters for $K_{p}, K_{i}$ and $K_{d}$ were chosen by a known PID tuning method.

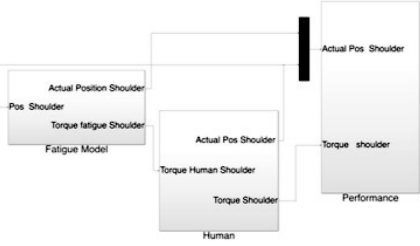

(a)

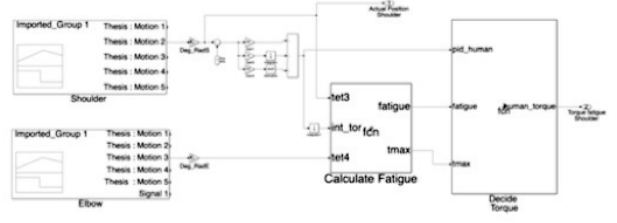

(b)

Fig. 1. (a) Human with fatigue model (b) fatigue model.

\section{Integration of Human Joint Fatigue and Exoskeleton}

Fig.(2)(a) presents the overall system for exoskeleton with human and Fig.(2)(b) presents the integration of the exoskeleton with the human fatigue model. There are three main blocks: PID, human-exoskeleton and performance block. Similar as in Section (2), both human and exoskeleton were controlled by using PID controller, and iterative approach is used to acquire the parameters.

'PID' block contains controller for human and exoskeleton. It also has two important blocks: Calculate Fatigue and Decide Assistive Torque block. 


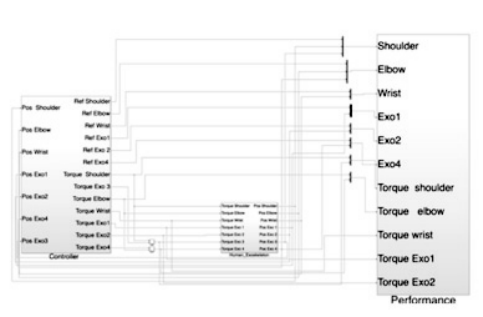

(a)

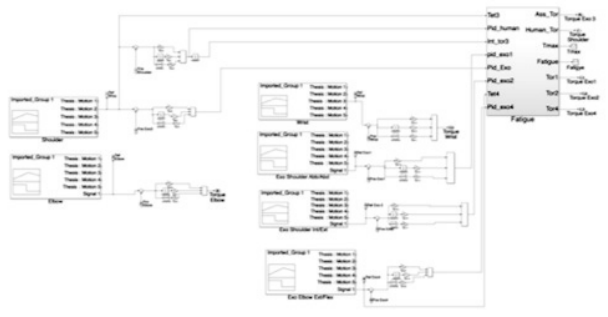

(b)

Fig. 2. (a) Human-exoskeleton system (b) Integration of exoskeleton and fatigue model.

Table 2. Parameters for (PID).

\begin{tabular}{cc}
\hline Parameter & Shoulder Joint \\
\hline Proportional & 10.0 \\
Integration & 1.0 \\
Derivative & 0.1 \\
\hline
\end{tabular}

In these two blocks, the algorithm for the activation process is presented. The first algorithm in 'Calculate Fatigue' block is presented as follows:

(i) To measure the human fatigue in joint level, there are three important information: maximum voluntary contraction of joint torque $\left(\tau_{M V C}\right)$, integrated joint torque $\left(\int \tau(u, \theta, \dot{\theta}, \ddot{\theta})\right)$ and angle for $\theta_{3}$ and $\theta_{4}$.

(ii) To calculate $\left(\tau_{M V C}\right)$, the formula is obtained from $\left[{ }^{6}\right]$. The formula is presented in Eq.(4). This value presents the maximum torque values for shoulder joint at $t=0$.

$$
\tau_{M V C}=\left(227.338+0.525 \theta_{3}-0.296 \theta_{4}\right) 0.2854
$$

(iii) Then, the fatigue could be measured by using Eq. 3. In this work, the value of fatigue rate $(\mathrm{k})$ is set to equal 1 . After applying fatigue equation, it is expected that the value of $\tau_{M V C}$ is reducing.

The algorithm in 'Decide Assistive Torque' block is presented as follows:

(i) A condition is set up to determine when human is fatigue. In this work, fatigue is identified when the maxmimum vountary contraction of the torque is reducing up to $80 \%$.

(ii) Once the joint fatigue is identified, an exoskeleton is sent torque support to maintain human in desired position.

if fatigue $<=0.8^{*} \tau_{M V C}$

then 


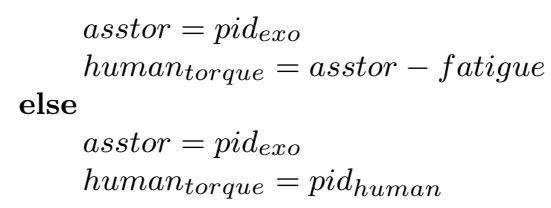

(iii) To maintain the position of human shoulder joint, the value of asstor is sent to an exoskeleton joint and human torque is sent to human shoulder joint.

In the next section, the results and discussion of the experiment is presented.

Table 3. Parameters for (PID).

\begin{tabular}{ccc}
\hline Parameter & Shoulder Joint & Exoskeleton Joint $\left(\theta_{3}\right)$ \\
\hline Proportional & 10.0 & 10.0 \\
Integration & 1.0 & 5.0 \\
Derivative & 0.1 & 0.5 \\
\hline
\end{tabular}

\section{Result and Discussion}

As mentioned in Section (2), the parameters for P, I and D for human shoulder were found by iteratively. The parameters are presented in Table (2). Fig. (3)(a) shows that human is able to maintain at desired position but, small oscillations occur at this joint. By looking at the oscillation in Fig. (3)(a) and Fig.(3)(b), we could said that, the oscillation is actually presenting the fatigue condition. This also could be seen in Fig. 3(c). As mentioned in Algorithm 1 of Section (3), fatigue is identified when the joint strength is reached to $80 \%$ of the $\tau_{M V C}$. Hence, the fatigue level is reducing but, when it reached to $80 \%$ of the $\tau_{M V C}$, it start to maintain at that value.

As mentioned earlier in Section 3, the parameters for PID controller is obtained with a known tuning PID approach and is presented as in Table (3). For the integration system (Exoskeleton-Human), the PID parameter for shoulder joint use the same values as in Table (2). These values were chosen to ensure that both system (human with joint fatigue model and exoskeleton-human model) are in the same condition. Hence, any changes that occur could be compared. 

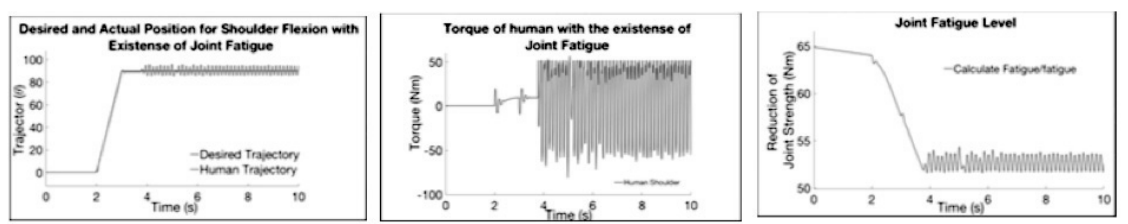

Fig. 3. Results of human with fatigue model: (a) Desired and actual trajectory of human (b) torque of human (c) fatigue level.
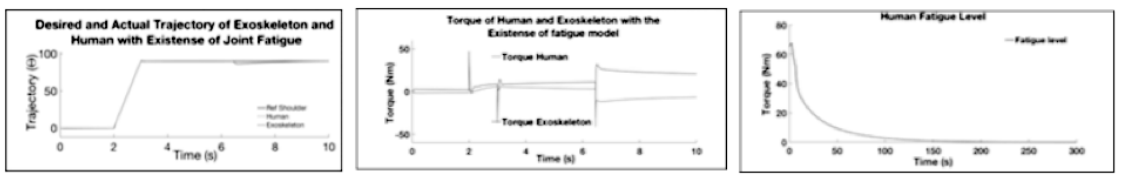

Fig. 4. Results of human fatigue with the existense of an exoskeleton: (a) Desired and actual trajectory of exoskeleton and human (b) torque of exoskeleton and human (c) human fatigue level.

Fig. (4)(a) shows the movement of human and an exoskeleton. The joint fatigue model is included in human model. As shown in Fig. (4)(a) when time reached at approximately to $6.70 \mathrm{~s}$, a small undershoot occur. There are two observations can be found here.

First, after shoulder joint is reached to 90 degree, shoulder joint is able to sustain at this trajectory approximately $3.70 \mathrm{~s}$. A small undershoot occur at $6.70 \mathrm{~s}$. At this point, it can be seen that shoulder joint is approaching at the maximum point of fatigue level and thus, it moves downward. Fig.(4)(a) also shows that the exoskeleton is moving downward following the human joint (the black circle), but eventually, both human and exoskeleton were moving to the desired trajectory back. From here, it shows that, exoskeleton is actually, supporting human to maintain at the desired point. Second observation is, human with an exoskeleton, took time to fatigue. This could be happened due to the support that is given by the exoskeleton from the beginning of the motion.

Fig.(4)(b) shows the torque required by the shoulder joint and exoskeleton joint. As shown here, an exoskeleton has high torque compared to shoulder joint, which shows that the exoskeleton is supporting the human upperlimb by providing an assistive torque. This could be seen clearly when the time is extended to $300 \mathrm{~s}$ (Fig.(5)). An exoskeleton is providing an assistive torque to ensure human could prolong doing the activities even tough, the strength of human joint has reach to $0 \mathrm{Nm}$ (Fig.(4)(c)). 


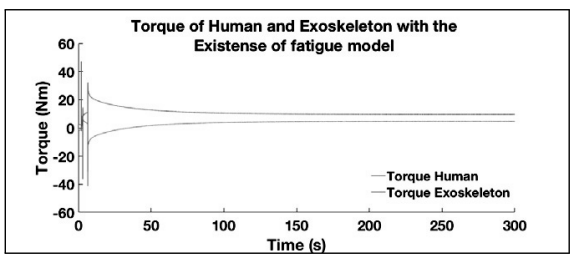

Fig. 5. Torque for exoskeleton and human.

\section{Conclusion}

Investigation into the impact of deploying an exoskleeton to compensate for human muscle fatigue have been presented using a virtual environment. A mechanism for monitoring and assessment of muscle fatigue has been presented. The information thus gathered allows the exoskeleton control to provide the required level of compensation to conteract the muscle fatigue. Future work will focus on incorporating fatigue compensation into the cotroller and further assessment of fatigue and corresponding compensation at various levels.

\section{Acknowledgments}

S. K. Ali is on study leave and supported financially by the Malaysia of Higher Education (MOHE) and Universiti Putra Malaysia.

\section{References}

1. F. P. Bonnet V., Colledani F. and S. N., J. Industrial Ergonomics 44, 475 (2014).

2. C. B. Xu W. and R. E., J. Control Engineering Practice 31, 63 (2014).

3. D. L. M. G. Carmichael and K. J. Waldron, Investigation of reducing fatigue and musculoskeletal disorder with passive actuators, in Proc. IEEE/RSJ Int. Conference on Intelligent Robots and Systems (MEMS'97), (Taipei, Taiwan, 2010).

4. B. F. Ma L., Chablat D. and Z. W., J. Industrial Ergonomics 39, 211 (2009).

5. B. F. Z. W. Ma L., Chablat D. and G. F., J. Virtual and Physical Prototyping, $1(2010)$.

6. M. R. Sakka S., Chablat D. and B. F., J. Human Factors Modelling and Simulation, 1 (2015). 